Trauma Berufskrankh 2012 · 14[Suppl 3]:331-334 DOI 10.1007/s10039-012-1846-0

Online publiziert: 25 . Mai 2012

(c) Springer-Verlag 2012
C. Meyer ${ }^{1} \cdot$ C. Kantelberg ${ }^{1} \cdot$ G. Szalay ${ }^{2} \cdot$ V. Alt ${ }^{2} \cdot$ R. Schnettler ${ }^{2}$

${ }^{1}$ Zentrum für Orthopädie und Unfallchirurgie, Klinikum Saarbrücken

${ }^{2}$ Klinik und Poliklinik für Unfallchirurgie, Universitätsklinikum Gießen und Marburg, Gießen

\title{
Periprothetische Femurfraktur nach Knietotalendoprothese
}

\section{Nagelosteosynthese}

\section{Hintergrund}

In Deutschland ist eine jährlich zunehmende Zahl primärer Knieendoprothesenimplantationen zu beobachten. Demzufolge steigt auch die Zahl notwendiger Knie-TEP-Revisions- (TEP: Totalendoprothese) und Wechseloperationen, wobei intra- und postoperative Komplika-

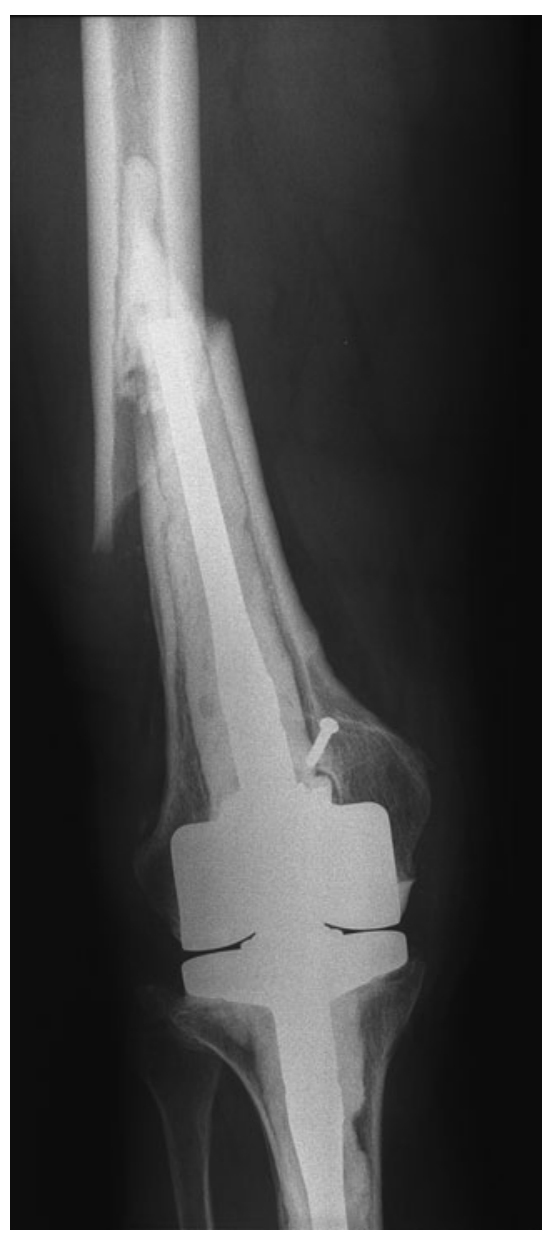

tionsraten von bis zu 31,0\% angegeben wurden $[2,3,7,10,11]$.

Periprothetische Femurfrakturen sind sowohl nach Hüft- als auch nach KnieTEP-Implantation problematisch zu behandeln. Darüber hinaus haben die Patienten, insbesondere nach Knie-TEPRevisionsoperation, in aller Regel bereits einen langen Leidensweg zu beklagen.

\section{Klassifikation der distalen}

Femurfrakturen bei einliegender Knieendoprothese

Für die Fraktureinteilung nach Knie-TEP wird derzeit der relativ grobe Score nach

Abb. $1 \varangle$ Periprothetische Femurschaftfraktur nach Revisionsknieendoprothetik, Typ 2 nach Rorabeck u. Taylor [9]
Rorabeck u. Taylor ([9], - Tab. 1) verwendet, da sich alternative Klassifikationen jeweils auf Oberflächenersatzendoprothesen beziehen und für die Situation nach Revisionsoperation nicht zu verwenden sind $[7,9]$.

\section{Therapieoptionen}

Zur Behandlung periprothetischer Femurfrakturen kommt bei stabiler Prothese meist die Plattenosteosynthese zum Einsatz, ggf. mit additiver Anlage einer Drahtcerclage. Auch die Anlagerung von Allografts wird in Einzelfällen durchgeführt. Beides sind Verfahren, die in aller Regel nur zu einer übungsstabilen Situation führen und keine Primärbelastung erlauben. Dennoch sind hohe Kom-

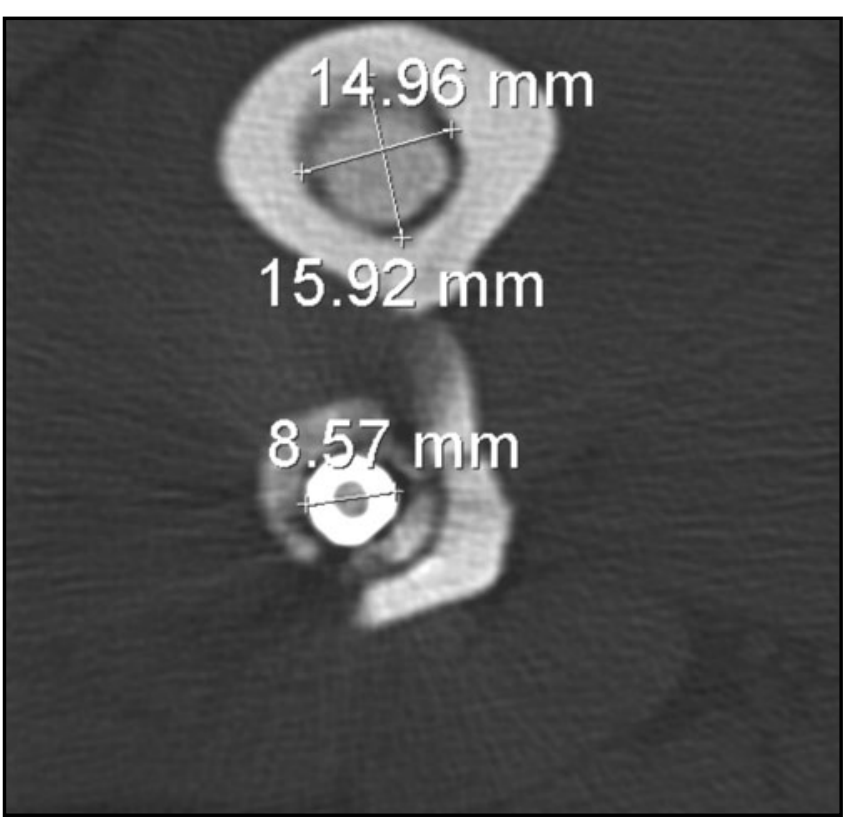

Abb. $2 \varangle$ Bestimmung der Fraktur- und Prothesendimensionen mittels Computertomogramm: Länge des proximalen Fragments, des Markraumund Prothesendurchmessers 


\section{Hätten Sie's gewusst?}

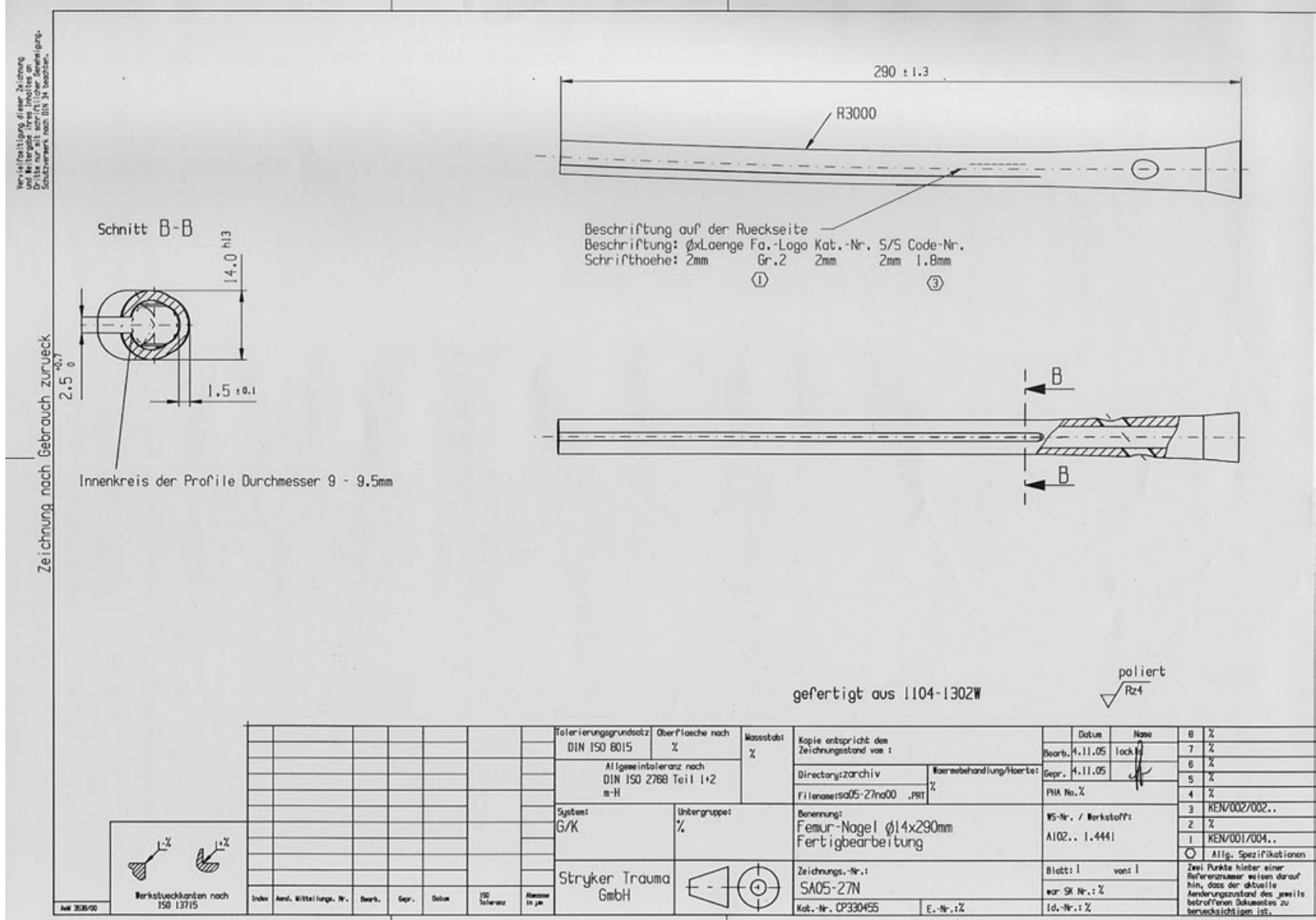

Abb. $3 \Delta$ Technische Zeichnung zur Herstellung des Sonderimplantats

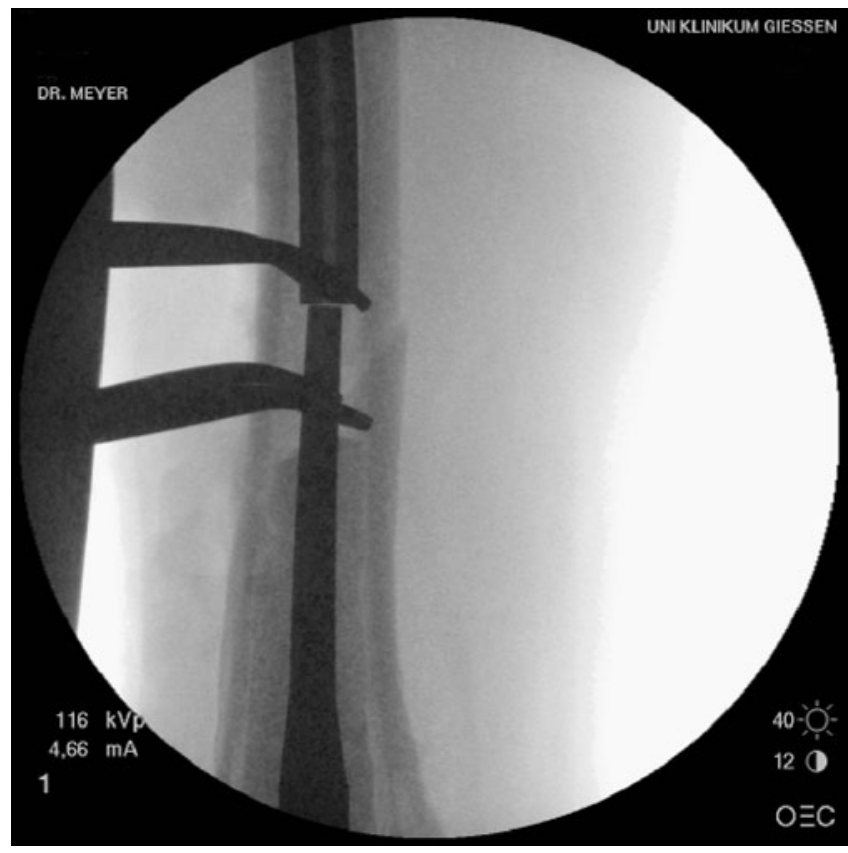

plikationsraten, in erster Linie eine hohe Pseudarthroserate, beschrieben. Bei gelockerter Endoprothese ist der Prothesenwechsel indiziert $[1,4,7,8,9,10,11,12,15]$.

Das Operationsverfahren der In-situKopplung der einliegenden Hüft- oder Kniegelenkendoprothese mit einem intramedullären Kraftträger stellt eine weitere Behandlungsalternative dar und kann in geeigneten Fällen durch Verlängerung der Prothese zu einer primären Belastungsstabilität in Verbindung mit einer zuverlässigen kallösen Frakturheilung führen [5, $6,13,14]$. Hierzu sind eine exakte präoperative Planung mit Größenbestimmung des Prothesenstiels, der Länge und des Durchmessers des Schaftfragments sowie die individuelle Herstellung eines Sonderimplantats erforderlich.

Abb. $4<$ Intraoperat ve Bildwandlerdarstellung des Dockingmanövers

\section{Fall und Methode}

Es wird über einen 59-jährigen Patienten berichtet, der nach berufsgenossenschaft- 


\begin{tabular}{|c|c|}
\hline Тур & Charakteristika \\
\hline 1 & $\begin{array}{l}\text { Undislozierte Fraktur } \\
\text { Stabile Prothese }\end{array}$ \\
\hline 2 & $\begin{array}{l}\text { Dislozierte Fraktur } \\
\text { Stabile Prothese }\end{array}$ \\
\hline 3 & $\begin{array}{l}\text { Undislozierte oder dislozierte Fraktur } \\
\text { Gelockerte Prothese }\end{array}$ \\
\hline
\end{tabular}

lich versichertem Unfallereignis eine Kreuzbandruptur erlitten und in der Folge eine posttraumatische Arthrose entwickelt hatte. Es folgte die Implantation eine Knie-TEP, welche aufgrund eines Infekts mehrfach revidiert wurde, einschließlich TEP-Ausbau und sekundärer Implantation einer Revisions-TEP. Im Rahmen der BGSW (berufsgenossenschaftliche stationäre Weiterbehandlung) stürzte der Mann während der Physiotherapie und zog sich eine periprothetische Femurfraktur zu (• Abb. 1).

Nach radiologischer Diagnostik wurden die exakten Längen und Durchmesser mittels CT-Untersuchung (CT: Computertomographie) bestimmt (ब Abb. 2). Auf Basis dieser Daten wurde ein geschlitzter Hohlnagel mit Verriegelungsmöglichkeit der entsprechenden Länge (2-3 cm Überlappung mit dem Prothesenstiel) und passendem Innendurchmesser (entsprechend dem Durchmesser des Prothesenstiels nach $2 \mathrm{~cm}$ ) angefertigt (D Abb. 3).

Am 5. Tag nach dem Trauma erfolgte die operative Versorgung durch offene Freilegung der Fraktur, Abstrichentnahme, Befreiung des Prothesenstiels auf einer Strecke von etwa $4 \mathrm{~cm}$ von Knochenzement, offenem Aufbohren des Schaftfragments und Einbringen des geschlitzten Hohlnagels. Dieser wurde unter Sicht- und radiologischer Kontrolle dem Prothesenstiel übergestülpt und mittels wohldosierter Hammerschläge stabil mit der Prothese verklemmt (• Abb. 4). Nach proximaler Verriegelung über das Zielgerät wurde der Eingriff mit dem schichtweisen Wundverschluss beendet.

Postoperativ wurde der Unfallverletzte unter schmerzadaptierter Belastungssteigerung bis zur Vollbelastung mobilisiert. Bei regelrechtem klinischem Verlauf zeigten die radiologischen Kontrol-

Trauma Berufskrankh 2012 - 14[Suppl 3]:331-334 DOI 10.1007/s10039-012-1846-0

C) Springer-Verlag 2012

\section{Meyer $\cdot$ C. Kantelberg $\cdot$ G. Szalay $\cdot$ V. Alt $\cdot$ R. Schnettler Periprothetische Femurfraktur nach Knietotalendoprothese. Nagelosteosynthese}

\section{Zusammenfassung}

Periprothetische Femurfrakturen nach Revisionskniegelenkendoprothetik werden mit zunehmender Häufigkeit beobachtet. Ein 59-jähriger Patient zog sich nach einer solchen in der Anschlussheilbehandlung im Rahmen eines Sturzes bei der Physiotherapie eine periprothetische Oberschenkelschaftfraktur zu. Die Behandlung erfolgte nach exakter präoperativer Planung mittels computertomographischer Messung der Größen- und Längenverhältnisse sowie Herstellung eines individuellen Implantats durch offene Reposition und Aufpfropfen eines Femurnagels auf den Prothesenstiel. Der Pa- tient wurde postoperativ unter schmerzadaptierter Vollbelastung mobilisiert, nach 12 Wochen war es zur knöchernen Konsolidierung bei schmerzfreier Vollbelastung gekommen. Die In-situ-Kopplung einer Endoprothese mit einem geschlitzten Hohlnagel stellt eine wertvolle Behandlungsoption bei periprothetischen Frakturen dar.

\section{Schlüsselwörter}

Knieprothese - Periprothetische Fraktur . Präoperative Planung · Marknagel · In-situ-Kopplung

\section{Periprosthetic femur fractures after total knee arthroplasty. Nail osteosynthesis}

\section{Abstract}

Nowadays an increasing number of periprosthetic femoral fractures are observed after revision knee arthroplasty. A 59-year-old male patient who underwent revision knee arthroplasty fell down in the rehab centre and suffered a periprosthetic femoral fracture. After exact preoperative CT planning and measurement of the ratio and linear dimensions a custom-made implant was produced and engrafted on the prosthesis stem accompanied by open reposition. Postoperatively the patient was mobilized with pain-relat- ed weight-bearing, and after 12 weeks osseous consolidation and pain-free full weightbearing were observed. In situ coupling of an endoprosthesis with a slotted hollow nail represents a valuable treatment option for periprosthetic fractures.

\section{Keywords}

Knee prosthesis - Periprosthetic fractures . Preoperative planning $\cdot$ Intramedullary nail . In situ coupling len eine deutliche Kallusbildung mit knöcherner Konsolidierung nach 12 Wochen (• Abb.5).

\section{Diskussion}

Die Behandlung periprothetischer Femurfrakturen nach Kniegelenkendoprothese ist ausgesprochen problematisch und komplikationsträchtig $[3,7,10,12]$. Die klassischen Prinzipien der Verriegelungsnagelosteosynthese, die nach Oberflächenersatz in der Regel angewendet werden, können nach Revisionsendoprothetik bzw. bei achsgekoppelter Prothese mit intramedullärer Verankerung nicht eingesetzt werden. Bei der Stabilisierung mittels Plattenosteosynthese ist die siche- re Fixierung des verwendeten Implantats am distalen Fragment problematisch. Des Weiteren wird aufgrund der biomechanischen Nachteile der extramedullären Implantatlage lediglich Übungsstabilität erreicht.

Das Operationsverfahren der In-situVerlängerung der einliegenden Prothese kombiniert den Vorteil der stabilen intramedullären Osteosynthese mit dem Erhalt der intakten TEP. Der operative Eingriff ist im Vergleich zum Prothesenwechsel weniger belastend, die erreichte Stabilität ist aus der klinischen Erfahrung der einer Plattenosteosynthese deutlich überlegen.

Trotz der Kompromittierung der Durchblutung des Knochens (offenes Operationsverfahren bei bereits beein- 

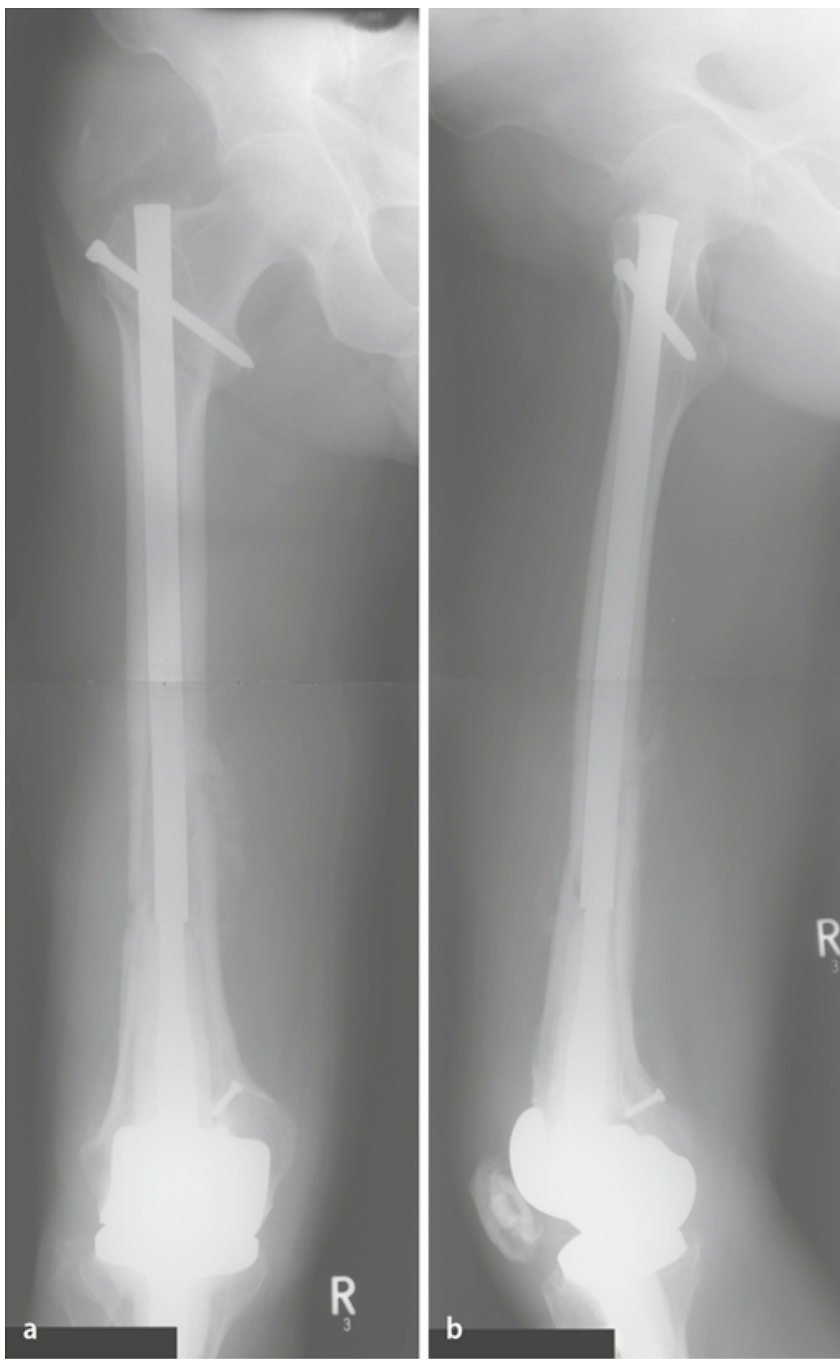

Abb. 5 Knöcherne Konsolidierung der Fraktur

trächtigter intramedullärer Zirkulation) wird regelmäßig eine zeitgerechte kallöse Konsolidierung der Fraktur erreicht [5, $6,13,14]$.

Die Indikation zur In-situ-Verlängerung einer Endoprothese wird unter folgenden strengen Kriterien gestellt [5, 6]:

- Frakturen des Typs 1 oder 2 der Rorabeck-Klassifikation [9], d. h. stabile Prothese,

- geeignete Dimensionen des proximalen Femurfragments zur Aufnahme eines passenden großvolumigen $\mathrm{Na}$ gels (ausreichender Markraumdurchmesser) sowie des Prothesendurchmessers,

- konisch zulaufende Spitze des einliegenden Prothesenschafts, die das Aufstülpen eines kurzen geschlitzten Verriegelungsnagels erlaubt (exakte präoperative Planung mittels CT bzw.
Überprüfung am identischen Prothesenmodell),

- Ausschluss einer Prothesenlockerung oder fortbestehenden Protheseninfektion sowie einer symptomatischen Koxarthrose,

- ausführliche präoperative Aufklärung über die Behandlungsmethode, die ein vom Standard der operativen Versorgung abweichendes Verfahren darstellt, einschließlich des Einsatzes eines sog. Custom-Made-Implantats.

\section{Fazit für die Praxis}

Die In-situ-Verlängerung der einliegenden Endoprothese mittels sonderangefertigem Marknagel stellt unter Berücksichtigung der Indikationskriterien eine wertvolle Behandlungsoption dar.

\section{Korrespondenzadresse}

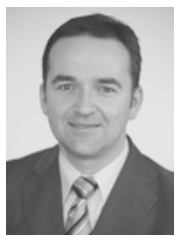

PD Dr. C. Meyer

Zentrum für Orthopädie und Unfallchirurgie, Klinikum Saarbrücken, Winterberg 1, 66119 Saarbrücken chmeyer@klinikumsaarbruecken.de

Interessenkonflikt. Der korrespondierende Autor gibt für sich und seine Koautoren an, dass kein Interessenkonflikt besteht.

The supplement containing this article is not sponsored by industry.

\section{Literatur}

1. Ayers DC (1997) Supracondylar fracture of the distal femur proximal to a total knee replacement. AAOS Instr Course Lect 46:197-203

2. Heck DA, Melfi CA, Mamlin LA et al (1998) Revision rates after knee replacement in the United States. Med Care 36(5):661-669

3. Hopf C, Hopf T, Rompe JD (1996) Behandlungskonzepte von Femurfrakturen nach totalendoprothetischem Ersatz des Hüft- oder Kniegelenks: intra- oder extramedulläre Stabilisierung? Unfallchirurg 99:3137

4. Jabczenski FF, Crawford M (1995) Retrograde intramedullary nailing of supracondylar femur fractures above total knee arthroplasty. J Arthroplasty 10:95101

5. Meyer C, Alt V, Schroeder L et al (2007) Treatment of periprosthetic femoral fractures by effective lengthening of the prosthesis. Clin Orthop Relat Res 463:120-127

6. Meyer C, Alt V, Heiss C et al (2011) Marknagelung periprothetischer Femurfrakturen nach Revisions-Knietotalendoprothese. Unfallchirurg 114:241-247

7. Mittlmeier T, Stöckle U, Perka C, Schaser K-D (2005) Periprothetische Frakturen nach Knietotalendoprothetik. Unfallchirurg 108:481-496

8. Probst A, Schneider T, Hankemeier S, Brug E (2003) Der Prothesennagel - primär belastungsstabiles Implantat bei peri- und subprothetischen Frakturen. Unfallchirurg 106:722-731

9. Rorabeck CH, Taylor JW (1999) Periprosthetic fractures of the femur complicating total knee arthroplasty. Orthop Clin North Am 30:265-277

10. Rolston LR, Christ DJ, Halpern A, O'Connor PL, Ryan TG, Uggen WM (1995) Treatment of supracondylar fractures of the femur proximal to a total knee arthroplasty. J Bone Joint Surg Am 77-A:924-931

11. Rupprecht $M$, Großterlinden L, Barvencik F et al (2008) Periprothetische Femurfrakturen. Unfallchirurg 111:812-820

12. Su ET, DeWal H, Di Cesare PE (2004) Periprosthetic femoral fractures above knee replacements. J Am Acad Orthop Surg 12:12-20

13. Tanaka Y, Kobayashi T, Ohashi M et al (2007) A new operative procedure using a Küntcher nail for a periprosthetic supracondylar femoral fracture after revision total knee arthroplasty: a case report. Knee 14:59-62

14. Wenda K (2002) In-situ-Kopplung eines hohlen Marknagels mit einer gestielten Knieprothese bei periprothetischer Fraktur. Unfallchirurg 105:19-22

15. Wick M, Müller EJ, Muhr G (2001) Suprakondyläre Femurfrakturen bei Knieendoprothesen. Unfallchirurg 104:410-413 